\title{
A Knowledge-Based Approach Towards Automated Manufacturing- Centric BIM: Wood Frame Design and Modelling for Light-Frame Buildings
}

\author{
Mahmud Abushwereb ${ }^{1}$, Hexu Liu ${ }^{2} *$, and Mohamed Al-Hussein ${ }^{3}$ \\ ${ }^{1}$ MSc candidate, Department of Civil and Environmental Engineering, University of Alberta \\ ${ }^{2}$ Assistant Professor, Department of Civil and Construction Engineering, Western Michigan \\ University \\ ${ }^{3}$ Professor, Department of Civil and Environmental Engineering, University of Alberta \\ *Corresponding author's e-mail: hexu.liu@wmich.edu
}

\begin{abstract}
Building information modelling (BIM) technology has the potential to improve communication among multiple stakeholders and to streamline construction projects. In order for the BIM model to be fit for use in the construction field generally and in modular construction projects specifically, it needs to be designed with sufficient construction details. However, in current practice, this requirement necessitates substantial manual modelling efforts, which limits the use of BIM in the construction field. In this context, the objective of this research is to automate BIM of construction details for modular construction (i.e., manufacturing-centric BIM) with a focus on the woodframing design and modelling processes. Specifically, this paper presents a portion of the research undertaken at the University of Alberta to develop FrameX, an Autodesk Revit add-on under development for the purpose of automating the framing design of light-frame wood structures. It represents a rule-based modelling approach that is capable of analyzing and designing building frames automatically in accordance with building codes, transportation regulations for modular components, and industry-wide best practices. Various best practice scenarios described in this paper represent ways the industry is seeking to reduce the material, time, and effort required to manufacture prefabricated building panels. A case study is presented to demonstrate the effectiveness of the rule-based modelling approach and the prototyped system, FrameX. The results reveal that the prototype system, FrameX, can automatically output manufacturing-centric BIM model and shop drawings in accordance with formalized rules, to assist field specialists from the outset of a given construction project.
\end{abstract}

\section{KEYWORDS}

Building Information Modelling (BIM); Automated Design and Modelling; Modular construction; Light-Framed Wood Buildings; Design for Manufacturing \& Assembly; Knowledge-based approach 


\section{INTRODUCTION}

Modular and off-site construction has changed the construction industry by creating a safer construction environment for labourers, for instance, and decreasing the probability of the risks involved in working at dangerous heights (Li, 2019). Additionally, it provides opportunities for employing automation in construction to improve efficiency, capitalizing on a user-friendly working environment in a factory setting (Liu, 2018). However, the prerequisites for construction automation are precise and well-made construction plans (Altaf, 2018). In this regard, Building Information Modelling (BIM) offers a promising solution to informed decision-making with regards to the construction plan. The information provided by the client could be translated by the designers to the benefits of the construction process using BIM solutions. Designers' working time can be decreased through the use of BIM technology in the design phase that ensures the incorporation of sufficient details in the construction drawings (Azhar, 2011). Besides, BIM has the potential of dealing with continuous design changes during the project with a high level of accuracy and up to date information must be easily and readily available. Plans can thus be developed for the manufacturing process such as the layout of production stations inside the factory, as well as the installation plan at the construction site (Alwisy, 2010).

However, in order for the BIM model to be fit for use in the construction field generally and in modular construction projects specifically, it needs to be designed with sufficient construction details. In current practice, this requirement necessitates substantial manual modelling efforts and trades' know-how regarding off-site construction processes, which limits the use of BIM in the construction field. For instance, in order to transport the building from the factory to the site, the building gets divided into modular units of the appropriate dimensions and weights to obey jurisdictional regulations for road transportation. When modelling the details for off-site construction projects, we have to rely on tacit knowledge and substantial manual modelling efforts to develop the BIM model. Industry professionals have come to realize that creating the BIM model manually is time-consuming and error-prone comparing to automating the process of building an efficient model. BIM also suffers limitations due to a lack of rules for modular construction, such as regulations for transportation of the building to the site. Currently, industry best practices are not tailored to using BIM. For example, framing design rules must be controlled by the user. All these specifications add a significant level of detail to the BIM model and increase its complexity.

Hence, the objective of this research is to automate BIM of construction details for modular construction (i.e., manufacturing-centric BIM) with a focus on the wood-framing design and modelling processes. Specifically, this paper presents a portion of the research undertaken at the University of Alberta to develop an Autodesk Revit add-on, FrameX, under development for the purpose of automating the framing design of light-frame wood structures. It represents a rule-based modelling approach that is capable of analyzing and designing building frames automatically in accordance with building codes, transportation regulations for modular components, and industrywide best practices.

\section{DESIGN FOR MANUFACTURING AND ASSEMBLY}

A variety of framing cases in the modular construction industry are formalized in this research. Shipping walls are the main focus of this paper as one of these complicated framing cases that need to be developed for a more flexible application. Shipping walls are temporary walls that need 
be installed for the purpose of transporting the modules to its final destination. The primary shipping regulations will vary depending on the location in Canada and the USA. Each region allows a maximum size and weight of modules. During the transportation of the building, sizes vary in height, length, and width of modules to be shipped smoothly without problems. Exceeding these requirements demands different permissions to be shipped to the final location. In addition, it must look like a closed box to prevent any wind and water complications. The problem, in this case, is how to design a temporary wall, which is used only during the transportation of a modular building and have it added to the BIM model automatically without affecting the work productivity by delaying the project. The shipping studs must be spaced a maximum of 24 inches-on-center to follow the building code and to use the minimum number of wasted studs. These extra framing components would be wasted and need to be considered as part of the total project cost; for example, large openings in between modules. The framing of these shipping walls may have a significant effect on the cost. Framing the exterior walls must be done in accordance with design rules and with no gap openings to comply with transportation regulations. Extra framing elements would be added just for transportation purposes.

\section{METHODOLOGY}

To achieve the objective, this research proposes a knowledge-based approach towards automating manufacturing-centric BIM with a focus on wood-frame design and modelling for light-frame buildings. These framing cases were an output of working on practical modular construction projects. Specifically, this paper presents the development of FrameX, a project that is being undertaken at the University of Alberta. FrameX is an application created with the intent to automate the process of drafting the structural members in light-frame wood construction. The prototype application performs the wood design on the platform construction method and generates shop drawings with quantity take-off automatically according to the BIM model. This reduces the design and drafting time and increases the productivity of the designer, with fewer errors (Liu, 2017). Using this method minimizes the wasted material and enhance the efficiency of labors during the construction process which is part of the advantages of using BIM technology (Liu, 2017). The objective of this paper is to prove the concept of using a BIM add-on to implement, automatically, the creation of shipping walls for the transportation of modular building components in the modular and off-site construction industry.

Figure 1 presents the proposed methodology of FrameX in analyzing the BIM model to create shop drawings for the structural components of the light, wood-framed building including the shipping walls. FrameX works for light-frame buildings where they often do not have designed wood columns to transfer the load of the building, and instead have vertical studs for this purpose. In the case where an opening is more than nine feet wide, the design might include designed columns on either side of the opening, which must be approved by a structural engineer. The logic of this application design follows the building code design rules for the spacing between structural components, connections, and framing around openings as illustrated in Figure 2. The attributes that need to be considered when framing a panel in offsite construction are represented and would be programmed as one function of classes that applies the design rules of the building code to join multiple walls with shipping walls (Liu, 2017). FrameX was created following the framing design rules in the building code, but lacked the flexibility to accommodate various framing scenarios encountered in the offsite industry specifically. FrameX was tested by modular construction 
companies and it was discovered, in some cases related to framing, that best practices need to be developed.

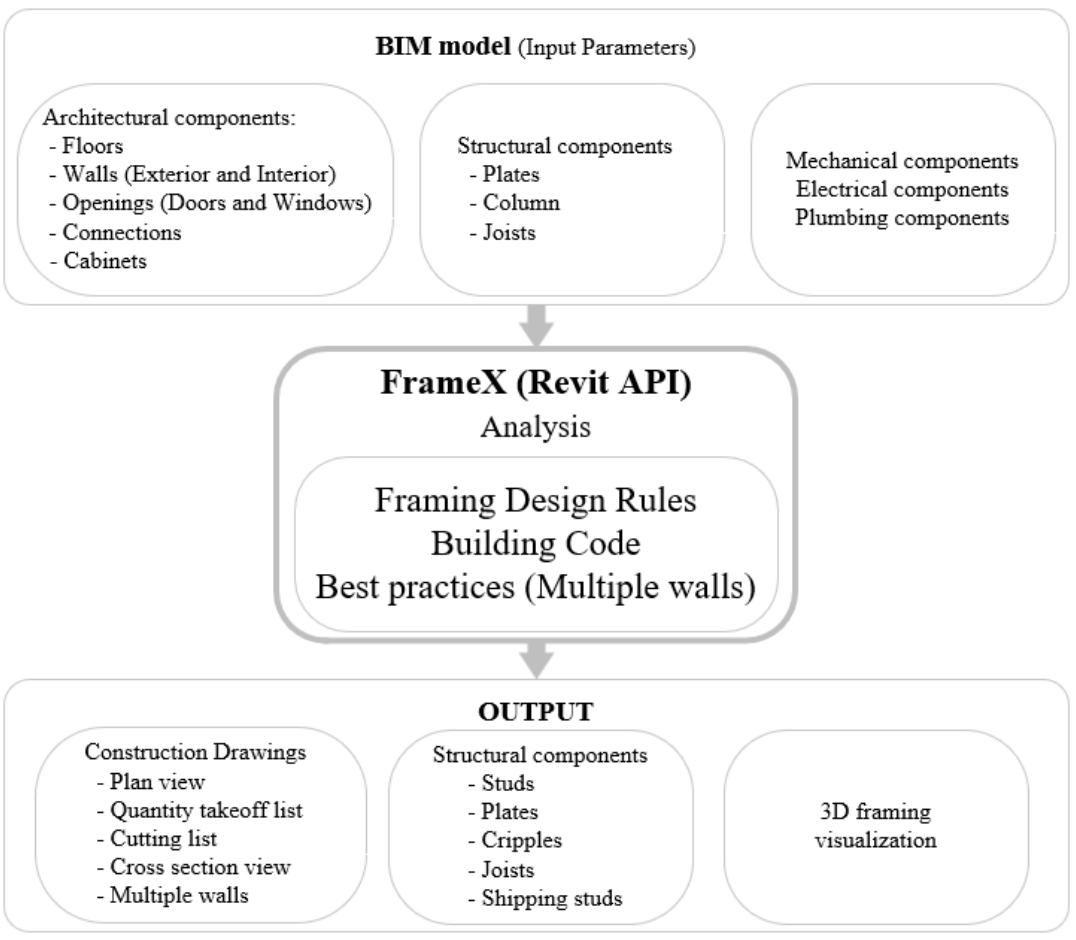

Figure 1. Proposed FrameX methodology

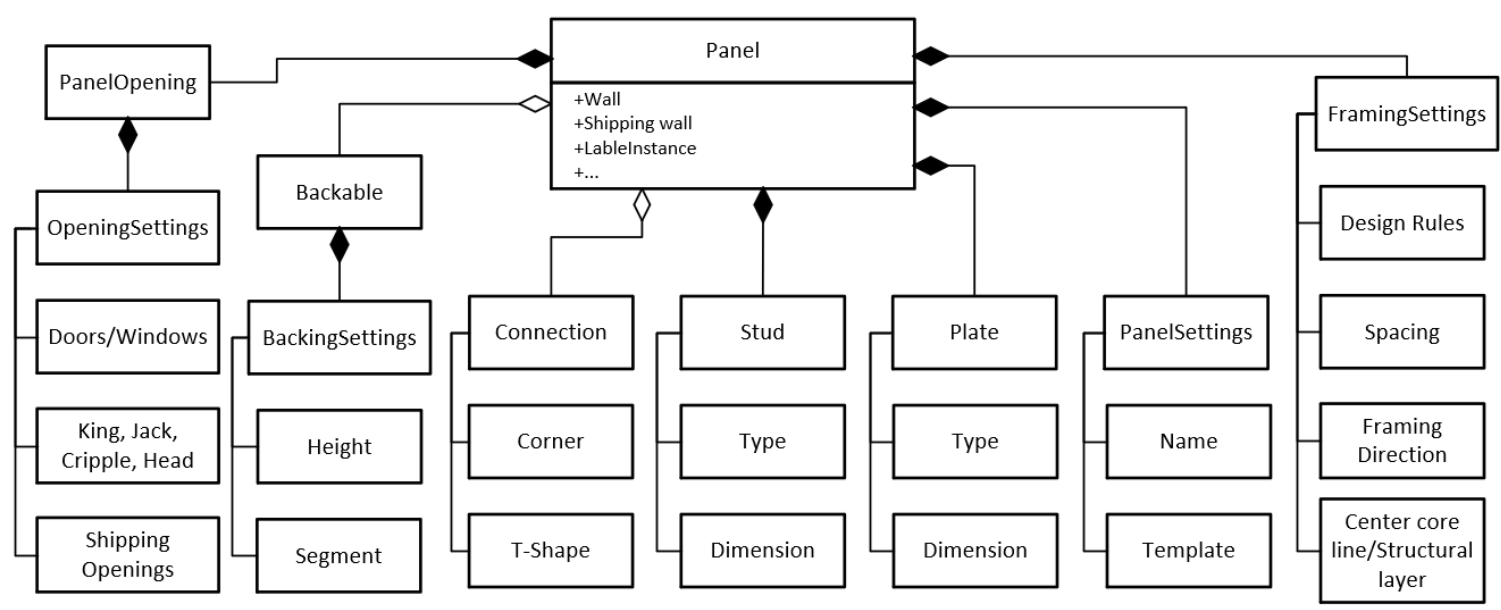

Figure 2. Structural framing design parameters and attributes for panel

The flowchart in Figure 3 illustrates the idea of how to use the multi-panel concept to create shipping walls. It aims to achieve the creation of multiple walls together with the shipping walls as it assembled in the manufactory. The limitation of this method is the top and bottom horizontal plate length and type. Long walls sometimes should go with the same dimensional lumber along the multi-panels. However, there is no problem with using different size of studs with different spacing. 
The software identifies the wall's attributes such as the length, height, thickness and openings as illustrated in Figure 2. It applies the framing design rules with the ability to choose the joined walls to create multi-panels as shown in Figure 3. It recognizes the wall's layers where it builds the structural elements in the center of the structural layer which is the core center line without affecting the exterior and interior layers. It determined the walls coordinates and frame the long wall accordingly. However, the target is to have the shipping studs aligned with the exterior core edges to enclose the modules with a sheathing layer. Figure 4 demonstrates the boundaries of the framing elements for the wall's top view. It starts framing from the core start point to endpoint according to the wall direction in the BIM model.

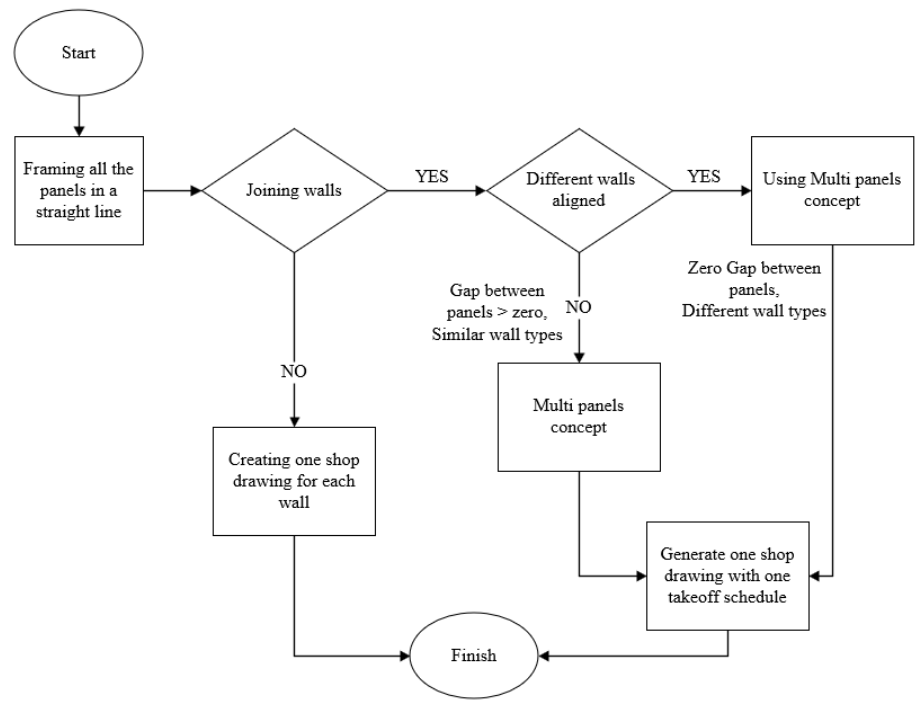

Figure 3. flowchart represents the logic to implement the method of multiple walls

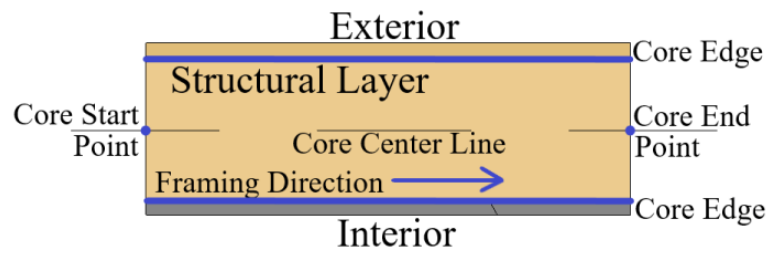

Figure 4. Top view for wall layers and boundaries

\section{CASE STUDY}

To validate the logic presented, a case study of wood-framed walls from a prefabricated home builder is examined. This case is presented after a couple of years of experience in testing the functionality of FrameX and ten modular construction projects that would have similar cases. For a single wall with a length of 60 feet, a comparison was made between framing the single wall manually using Autodesk Revit and using the prototype FrameX application. Table 1 shows how much time was required to frame one 60-foot long wall and create the shop drawings. Using the prototype application results in an 80 percent reduction in the amount of time required for this task. Next, the same comparison is undertaken for the framing of multiple walls and shipping walls with a total length of 60 feet. Table 2 shows the amount of time required to frame the multiple walls. FrameX required more than three minutes to accomplish the framing task, where the user 
had to make manual changes in Autodesk Revit to the original framing. The reason behind the long time process for the framing is that the application cannot frame through openings.

Table 1: Time required to frame one 60 -foot wall manually and by FrameX

\begin{tabular}{lcccc}
\hline 60 feet & Framing & Shop drawing & Quantity & Total \\
\hline Manual & $3: 25$ & $0: 37$ & $0: 31$ & $4: 33$ \\
Application & $0: 15$ & $0: 30$ & 0 & $0: 45$ \\
\hline
\end{tabular}

Table 2: Time required to frame 60-foot wall with shipping openings manually and by FrameX

\begin{tabular}{lcccc}
\hline 60 feet & Framing & Shop drawing & Quantity & Total \\
\hline Manual & $10: 17$ & $1: 02$ & $1: 25$ & $12: 44$ \\
Application & $1: 00$ & In progress & In progress & $2: 05$ (expected) \\
\hline
\end{tabular}

Table 2 presents that FrameX is not able to automatically perform framing through shipping openings. This process put a limitation on the functioning of creating full shop drawings. Therefore, this research is looking at how to add this feature to improve application performance. As illustrated in table 2 where this process is in progress on development to minimize the time taken to draft the structural components where the total time to fully automate framing the shipping walls would be expected to save eighty percent. The multiple walls and shipping walls in the above-mentioned case may be described as three walls aligned along one straight line that are built together as one assembly plus the shipping walls in-between as shown in Figure 5. The spacing for the standard exterior wall is 16 inches-on-center, and the shipping walls are framed with 24 inches-on-center stud spacing using less expensive studs to reduce the cost and the amount of material used.

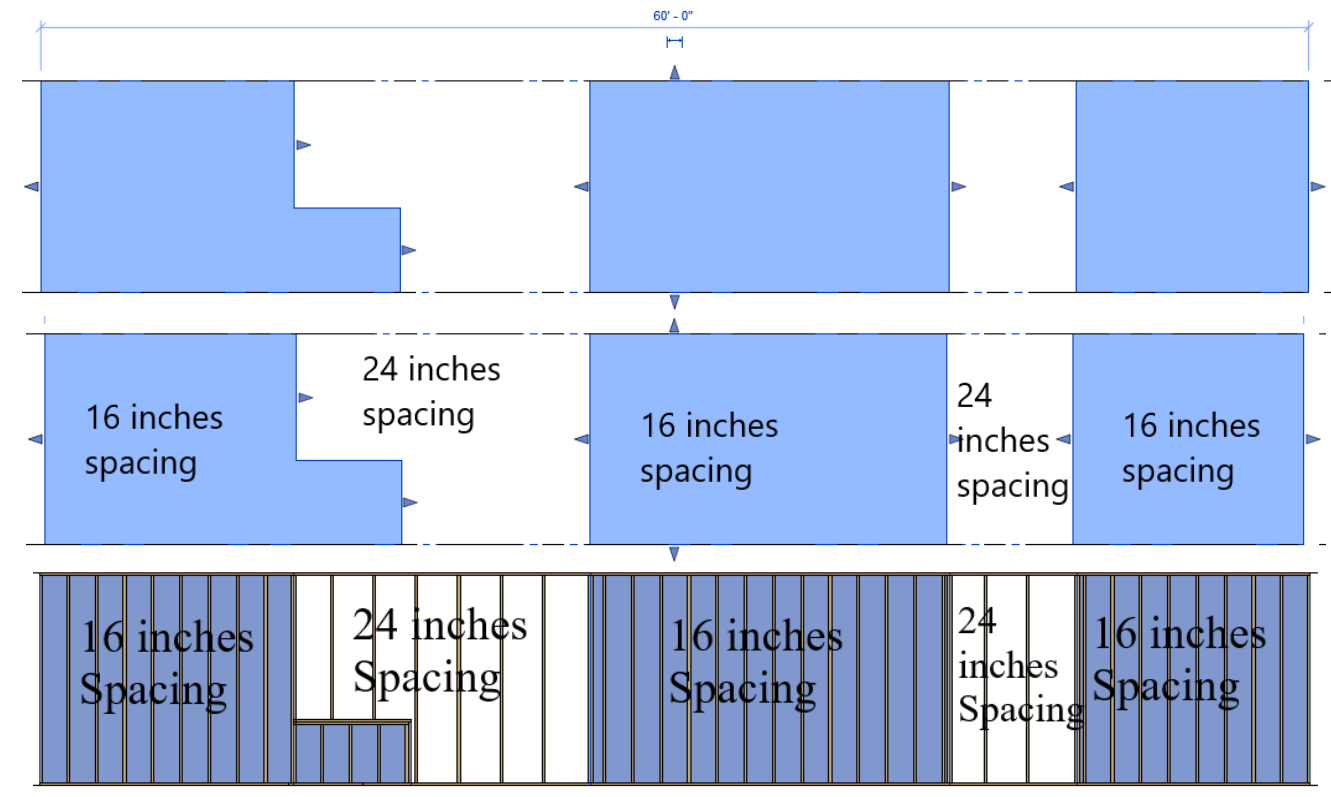

Figure 5. Stud Spacing on Multiple walls with shipping openings (Elevation view)

Revit lookup add-ins for checking the Revit API were used to determine the wall configuration as shown in Figure 5 (Snoop Objects >> Wall >> Geometry Objects Elements >> Face Array). This 
wall has more than the usual number of face plans due to its complexity. It has different planer face and edge elements where each one represents the points' coordinates. Each face has different points according to its geometry and location.

The developed logic identifies each wall in the system based on function and location. It identifies wall boundaries as shown in Figure 5, which are wall points representing the height and length. It is expressed in order according to the point location within the wall. Once the wall boundaries are recognized by the algorithm and first-order logic, every wall and shipping wall are sorted depending on the component characteristics such as length, height, and thickness based on the architectural BIM model.

\section{RESULTS AND DISCUSSION}

Automation of the framing process is one crucial component when the objective is to improve the productivity rate of drafters and designers. Users must apply manual changes to meet the regulations standards regarding the transportation of building modules. This research focuses on decreasing the number of manual changes required, which would lead to fewer errors in the drawings and fewer working hours. A comparison between manual and automated work was made to show the effectiveness of utilizing the prototype application FrameX. As shown in Figure 5, the application can determine the locations of boundaries of walls and areas and apply the framing accordingly with different framing scenarios.

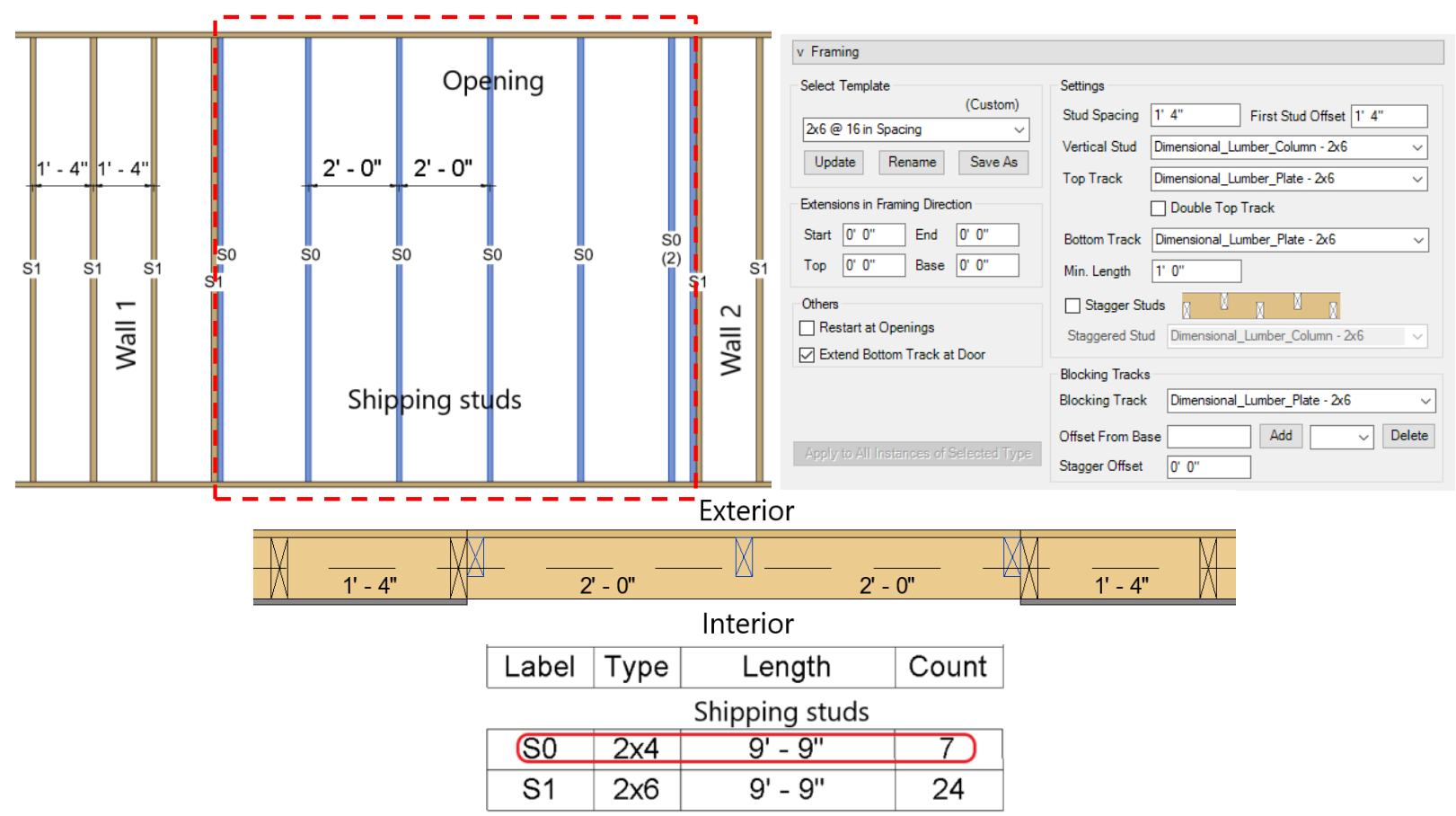

Figure 6. Result of this approach

Figure 6 represents the sample result that is expected as an output of this process and part of the software interface. It shows the locations of the shipping studs as highlighted. Also, shipping studs can be described with a unique member ID as S0 for example and the table shows the quantity of these elements. The goal of this case study is to automate the process of framing shipping walls, which are comprised of multiple panels with a gap for the shipping studs. The main wall will be 
framed according to user specifications. Creating a shop drawing automatically with these details is the objective in order to reduce the working time for the drafter. Therefore, this paper focuses on increasing the flexibility of the Revit add-on FrameX to accommodate scenarios specific to modular and off-site construction and to accommodate a higher level of complexity in framing applications. In the future, this research is looking towards reducing the time required for the framing of special framing cases by up to 80 percent.

\section{CONCLUSION}

This paper proposed a procedure to expedite the process of framing design using Autodesk Revit and creating shop drawings through the structural layer of the wall and exterior core edge. It presented findings for a particular framing case of multi-walls and shipping walls with varying spacing between vertical studs which is a common case in the industry of offsite construction. It is crucial for the industry to use developed applications to enhance drafters' productivity. Although the developed prototype, FrameX, works in normal circumstances of framing based on the overall framing rules, it will intelligently adapt different complicated scenarios. Applying the presented method to the application is still in progress, but it is expected to have a positive impact on the industry. It generally improves the drafting process at an early design stage of projects in terms of time and accuracy.

\section{ACKNOWLEDGEMENTS}

The authors would like to acknowledge the financial support of the Natural Sciences and Engineering Research Council of Canada (NSERC).

\section{REFERENCES}

Alwisy, A., Al-Hussein, M., and Al-Jibouri, S. H. (2012). "BIM Approach for Automated Drafting and Design for Modular Construction Manufacturing." Computing in Civil Engineering

Azhar, S. (2011). "Building information modelling (BIM): Trends, Benefits, Risks, and Challenges for the AEC industry." Leadership and Management in Engineering,

Manrique, J.D., Al-Hussein, M., Bouferguene, A., and Nasseri, R. (2015). "Automated generation of shop drawings in residential construction." Automation in Construction, 55, 15-24.

Altaf, M. S., Bouferguene, A., Liu, H., Al-Hussein, M., \& Yu, H. (2018). "Integrated production planning and control system for a panelized home prefabrication facility using simulation and RFID". Automation in Construction, 85, 369-383.

Liu, H., Singh, G., Lu, M., Bouferguene, A., \& Al-Hussein, M. (2018). "BIM-based automated design and planning for boarding of light-frame residential buildings". Automation in Construction, 89, 235-249.

Liu, H., Holmwood, B., Sydora, C., Singh, G., and Al-Hussein, M. (2017). “Optimizing multi-wall panel configuration for panelized construction using BIM.” Proceedings, 2017 International Structural Engineering \& Construction Conference (ISEC), Valencia, Spain, Jul. 24-29.

Li, X., Han, S., Gül, M., \& Al-Hussein, M. (2019). “Automated post-3D visualization ergonomic analysis system for rapid workplace design in modular construction". Automation in Construction, 98, 160-174.

Li, X., Han, S., Gül, M., Al-Hussein, M., \& El-Rich, M. (2017). “3D Visualization-Based Ergonomic Risk Assessment and Work Modification Framework and Its Validation for a Lifting Task". Journal of Construction Engineering and Management, 144(1), 04017093. 